\title{
ULTRASONIC MATERIAL PROPERTY DETERMINATIONS
}

\author{
Steven Serabian \\ University of Lowe 11 \\ Lowe 11, Massachusetts 01854
}

The use and potential offered by ultrasonic velocity and attenuation measurements to determine and/or monitor material properties is explored. The basis for such unique measurements along with examples of materials from a variety of industries will be presented.

\section{INTRODUCTION}

The ability of ultrasound to measure and/or monitor microstructure related properties has been established in the laboratory. A number of applications have been implemented for field use, however the full potential of such technologies has not been realized. Invariably one makes use of two of the propagational parameters of ultrasound - i.e., attenuation and velocity. The latter are intrinsic to the particular combination of material and processing under consideration. Absolute quantitative relations are usually unavailable, therefore empirical material property ultrasonic paràmeter correlations based upon qualitative reasoning are sought. In effect, a series of specimens containing the range of the material property of interest is necessary along with conventional destructive material property measurement schemes. obviously, such correlations are not universal and are only valid for the particular material and attendant processing being investigated. Correlation sensitivity is the prime factor that defines the accuracy and effectiveness of the generated correlation. Once the correlation has been established costly and time consuming conventional destructive evaluation procedures can be eliminated. In this paper a number of examples of correlations of significant material properties with ultrasonic parameters will be cited.

\section{VELOCITY BASED CORRELATIONS}

The original low pressure piping systems used by the utilities to transport gas were constructed of cast iron. Mechanical properties and susceptibility to corrosion renders cast iron undesirable for such an application. Economics precludes the total replacement 
by steel wraped pipes, thus one must resort to a gradual, scheduled replacement based upon a measure of quality. The determination of the necessary mechanical properties required the need for an extensive length of piping. This presented a costly as well as a limited number of pipe sections that can be evaluated during normal installation and maintenance programs. However, it is possible to trepan a $1-1 \frac{1}{2}$ " coupon without shutting down the piping system. The readily available large number of coupons would then present a greater sampling rate of the piping system. A program was initiated which would facilitate the use of the coupops for the determination of significant mechanical properties. (1) The latter along with other parameters such as soil condition, corrosion and road traffic activities are used to formulate pipe quality.

Industry wide practice consists of using the ASTM Talbot strip beam test as shown in Figure 1 to measure mechanical properties. The maximum deflection of the beam at fracture allows the measurement of the moduli of elasticity and rupture. Radiography was used to aid in the selection of the specimen location within any given pipe section to eliminate occasional structural anomalies such as voids and inclusions. Each beam specimen was characterized by velocity and attenuation profiles prior to the destructive phase, see Figure 2. The attenuation proved to be too sensitive to microstructure even at low frequencies. However, the velocity provided a consistent value for each specimen yet sufficient differences between specimens. It is for these reasons that velocity was selected as the ultrasonic parameter to be used in the correlations with material properties. The velocity measurement scheme involves locating the transmitted $R-F$ pulse on a calibrated time scale. The calibration is provided by using specimens with a range of thickness and a known velocity.

There is quantitative substantiation of a velocity-mechanical properties correlation provided by,

$$
\begin{aligned}
v_{L}^{2} & =\frac{E_{d}}{\rho} \frac{(1+\delta)}{(1+2 \delta)(1-\delta)} \\
& =\frac{E_{d}}{\rho} f(\delta)
\end{aligned}
$$

Where Ed is the dynamic modulus, $\hat{f}$ is the density and $\delta$ is Poisson's ratio. By noting that $f(\delta)$ is essentially unity for a brittle material such as cast iron and that there is a direct relationship between Ed and the secant modulus $\left(E_{s}\right)$, therefore equation 1 becomes,

$$
\mathrm{V}_{\mathrm{L}}^{2} \cong \frac{\mathrm{E}_{\mathrm{s}}}{\rho}
$$


For the correlation $V_{L}$ and density are taken adjacent to the fracture point $; V_{I}$ from ${ }^{L}$ a display typified by Figure 2 and density by the water $L$ displacement technique from a small section of the strip.

Figure 3 indicates the secant modulus. The data spread is $\pm 1 \times 10^{6}$ psi. At the midpoint of the modulus range considered this amounts to \pm 128 and is within the error associated with conventional destructive measurement procedures.

The other property of interest was the modulus of rupture (R). In a brittle material,

$$
E_{S}=\frac{R}{\varepsilon_{T}}
$$

Where $\varepsilon_{T}$ is the strain to failure. Since $\varepsilon_{\mathrm{T}}$ can only be measured destructively it was necessary to seek an indirect relationship involving a property that is more readily available. One such property is hardness $(\mu)$ or,

$$
\varepsilon_{\mathrm{T}}=\mathrm{c} \mu
$$

where $C$ is a constant. Incorporating Equations 3 or 4 into Equation 2 yields,

$$
R \approx \rho v_{L}^{2} \mu
$$

The hardness was measured adjacent to the fracture point. The results of this correlation is shown in Figure 4. The error for a modulus of rupture determination is $\pm 5,500$ psi. Viewing this error at the midpoint of the $R$ range translates into $\pm 10 \%$ which is a creditable determination.

Once the beam specimen correlations were established all the necessary measurements of density, hardness and ultrasonic velocity can be obtained from the parallel faced coupons. The need for the costly removable of large pipe sections and the associated destructive evaluations are eliminated. Moreover, the coupons, because of greater availability provides a more realistic overall assessment of the quality of the piping system and can be used as a guide for the pipe replacement program.

Concrete is another material that falls into the brittle category and is therefore susceptible to the determination of strength related properties by velocity based measurements. (2) 
A variety of water to cement ratios as well as curing times were considered. The cylindrical specimens were used for measurement of compressive strength and modulus while step wedges were used for the assessment of attenuation. Figures 5 and 6 indicate the results of the destructive phase for the compressive strength and secant modulus, respectively. The influence of the curing time and the water to cement ratio is as to be expected; both increase the compressive strength and the secant modulus. Figure 7 displays the axial longitudinal velocity as a function of the curing time and the water to cement ratio. The similarities between the data of Figures 5 and 6 with Figure 7 suggests that a correlation exists between these properties and ultrasonic velocity. Figure 8 shows the compressive strength correlation where it is significant to note that notations involving composition and curing time are lost. The accuracy ( \pm 500 psi) is within that expected by conventional destructive means. Much the same remarks are in order for the secant modulus correlation display as Figure 9 .

Some interesting observations regarding the state of cure can be noted by the use of attenuation. The attenuation in concrete is too excessive to measure by noting the decay of a multiple order back reflection. In this case the slope of an amplitude thickness display obtained by the step wedge specimen proved to be a valid technique. A torque wrench was used in a drill press arrangement to maintain a constant transducer pressure at each step. (1I) Figure 10 shows a typical determination for a water to cement ratio of 0.5 . It can be seen that the attenuation is high during the initial phase of cure. An attenuation decrease occurs as the structure becomes more rigid thus less energy is expended to propagate the ultrasound.

Another example of characterizing a material by velocity measurements is graphite of aerospace applications. (3) Figure 11 indicates a data display and serves to illustrate the sensitivity for density; the dynamic modulus was measured by resonant techniques. It should be noted that a variety of grades and velocity measuring directions are involved. Such a density sensitivity suggests that transport phenomena are susceptable to velocity correlations. Figure 12 supports this notion for the case of electrical resistivity.

\section{ATTENUATION BASED CORRELATIONS}

Let us now turn our attention to the use of attenuation for the characterization of materials; in particular to the polycrystalIine state. The functional dependency of the average grain size $(\bar{D})$ and frequency (f) upon attenuation is shown in Figure 13 where $\overline{\Delta K}$ is the direction related average change in elastic pro-

$\overline{\mathrm{K}}$ perties of the grains encountered by the ultrasound. (4) In Rayleigh scattering the whole grain acts as a scattering unit. 
For phase scattering one must be cognizant that each portion of the ultrasonic beam contends with a discrete set of grains, thus producing a variety of phases at the detector. The summation of these individual phases constitutes the phase scattering loss. In diffusion scattering one is essentially concerned with mean free path considerations - i.e., how many grain boundries are encountered. The dependency of these loss mechanics upon grain size and frequency can be shown experimentally. Figure 14 displays typical data for a steel that has been heat treated to obtain a range of grain size. The deviation from linearity at the lower frequencies for the less attenuating specimens is due to transducer beam spreading or diffraction losses. Appropriate corrections would, in effect, reduce each data point in this region to the linear extrapolated high frequency data. The slope of each attenuation - frequency display is the exponential frequency dependency $(n)$. Figure 15 shows the frequency exponent for a variety of materials with different grain sizes. (5) The spread in the data is primarily due to the fact that each specimen can have a range of grain size, thus more than one type of scattering mechanism is in effect.

By cross plotting the data of Figure 14 one can note the grain size dependancy (m) of attenuation. See Figure 16. Figure 17 shows such data for a number of materials and indicates that the grain size dependancy can range from zero to three. The data set is not capable of examining the diffusion type of loss $(m=-1)$.

Of importance for this discussion is the grain size dependency (m). Since for most engineering materials/structures the upper frequency limitation for propagation restricts scattering to the Rayleigh $(m=3)$ and phase $(m=2)$ loss mechanisms, thus attenuation is quite sensitive to grain size. One obvious use of this fact is the measurement of grain size. Figure 18 indicates the observed attenuation in a Timkin Alloy $(16-26-6)$ for a range of grain size. If the data is plotted in terms of the ratio of the average grain size to the dominate wavelength of a narrowband pulse of ultrasound, a single curve is obtained (Figure 19). This is in accordance with Roney's generalized scattering theory that allows one to consider attenuation in terms of a dimensionless parameter that obviates the need to consider both the grain size and wavelength individually. By making attenuation measurements at a variety of frequencies one can obtain an overall grain size determination.

The sensitivity of attenuation to grain size suggests the feasibility of using attenuation to measure any material property that is grain size dependent. Figures 20 and 21 indicate the

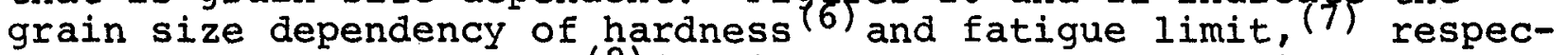
tively. The data of Low $(8)$ in Figure 22 shows the grain size dependency for strain in failure as well as fracture and yield stresses. As shown in Figure 23 Klinman(9) has utilized the grain size dependency of attenuation to determine transition temperature for a spectrum of carbon steels.

In addition to grain structure dependency attenuation is also 
influenced by other scatters such as porosity and inclusions of microscopic proportions. Such scattering losses contribute to the observed attenuation. Figure 24 attests to the sensitivity of attenuation to porosity in grain refined aluminum. (10) A uniform grain size throughout the specimen set assures that porosity is the dominate cause of the attenuation variations. The porosity, as verified in Figure 25, should effect the ultimate tensile strength and be measureable by attenuation.

Silicate inclusions are prevalent in 303 stainless steel and provides an example of microscopic inclusions acting as scatter sites. The specimens considered contained approximately the same grain size thus did not contribute to the observed attenuation variations. Inclusion counts were performed by a quantitative television microscope and were rated in accordance with length in six levels from 2 to 12 microns in 2 micron intervals. Using a weighting function of $\mathrm{n}^{2}$ to bring out the influence of the larger inclusions a measure of the inclusion severity was taken as $\sum^{2} n^{2} \mathrm{Sn}$ where $\mathrm{Sn}$ is the number of inclusions in the $\mathrm{n}$ th counting level. The results are shown in Figure 26.(12) As to be expected the higher frequencies present a greater sensitivity to the inclusion count. Such data can be used to quality grade the material.

Another example of inclusion produced attenuation is displayed in Figure 27. The structure is a large rotor forging used in large steam turbines. The center is the last to solidify, thus is the major recipient of the impurities. It is for this reason that a small borehole is cored out. The data shows the attenuation as a function of the axial length. The attenuation is measured by the decay in a multiple order bore refraction display and subsequently corrected for geometric effects originating from the curved surfaces encountered. It can be seen by the solid line that the initial measurement indicated an axial region that showed abnormally high attenuation. This was also verified by an attenuation measurement of the core material from this region as well as magnetic particle inspection of the bore surface. It was decided to subject the rotor to a minimal overboring operation. As evident by the dotted line display the attenuation decreased appreciably in the region of high inclusion count while the attenuation in other regions remained essentially unchanged. The latter lends credance to the notion that the high inclusion count was localized. While this example is highly qualitative it does serve to indicate that attenuation is susceptable to scattering losses due to microscopic inclusions.

\section{SUMMARY AND CONCLUSIONS}

Examples of the utilization of ultrasonic velocity and attenuartion for the measurement and monitoring of material properties have been presented. It is evident that a wide spectrum of activities in a number of industries are involved. It is significant to note that the measurement schemes and attendant instrumentation 
have, in general, minimal requirements of sophistication and complexity.

\section{REFERENCES}

1. S. Serabian and G. E. Lockyer, "Nondestructive Evaluation of Cast Iron Pipe" Univ. of Lowell Research Foundation, April 1971.

2. S. Serabian and J. Tsererides, "Property Measurements of Concrete", presented at annual ASNT meeting, 1972.

3. G. E. Lockyer, "Investigation of Nondestructive Methods for the Evaluation of Graphite Materials", Air Force Materials Laboratory, TR-65-113! 1965.

4. E. P. Papadakis, "Ultrasonic Attenuation Casued by Scattering in Polycrystalline Media", in Physical Acoustics (Volume IVB); W. P. Mason, Editor, $196 \overline{8}$.

5. S. Serabian, "Frequency and Grain Size Dependency of Ultrasonic Attenuation in Polycrystalline Materials", Brit. $\mathrm{J}$. of NDT, 22, 2, 69, 1980 .

6. R. W. Armstrong, "The Influence of Polycrystalline Grain Size on Several Mechanical Properties", Met. Trans. ASM, 44, 929, 1952 .

7. G. M. Sinclair and W. J. Craig, Trans. ASM, 44, 929, 1952 .

8. J. R. Low, "The Relation of Microstructure to Brittle Fracture", ASM, 1954.

9. R. Klinman, R. et al, "Ultrasonic Prediction of Grain Size, Strength and Toughness in Plain Carbon Steel", Material Evaluation, 38, 26, 1980 .

10. K. Chinnathambi and O. Prabhaker, "Quality Control of Aluminum Alloy Castings", Trans. Indian Institute Metals, 33, 4, 296, 1980 .

11. S. Serabian, "Influence of Attenuation Upon the Frequency Content of A Stress Wave Packet in Graphite", J. Acoust. Soc. Am., 42, 5, 1052, 1967.

12. S. Serabian, "Inclusion Count Determinations by ultrasonic Attenuation Measurements", Armco Steel Corp., Middletown, Ohio, Dec. 1976. 

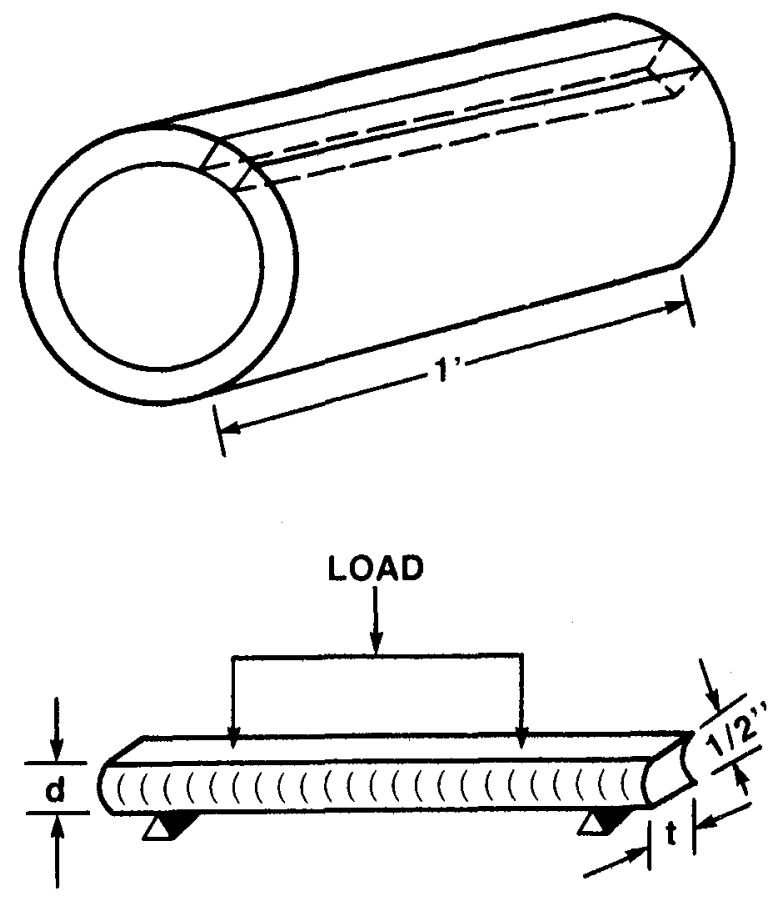

Fig 1 Talbot strip test.

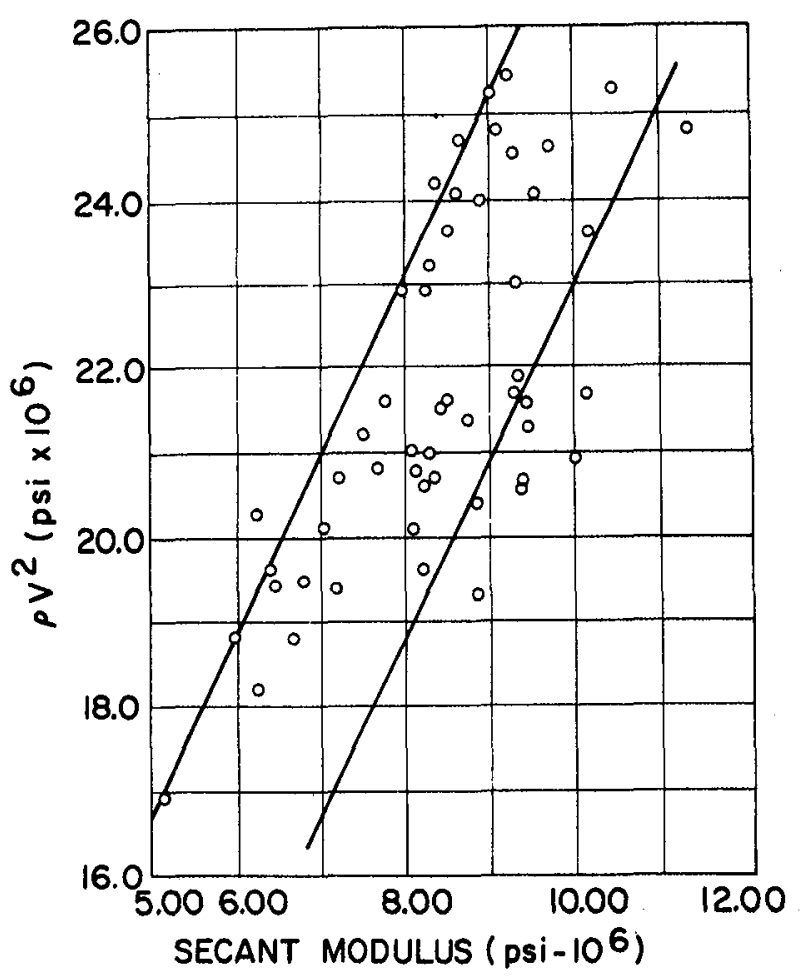

Fig 3 Secant modulus correlation in cast iron.
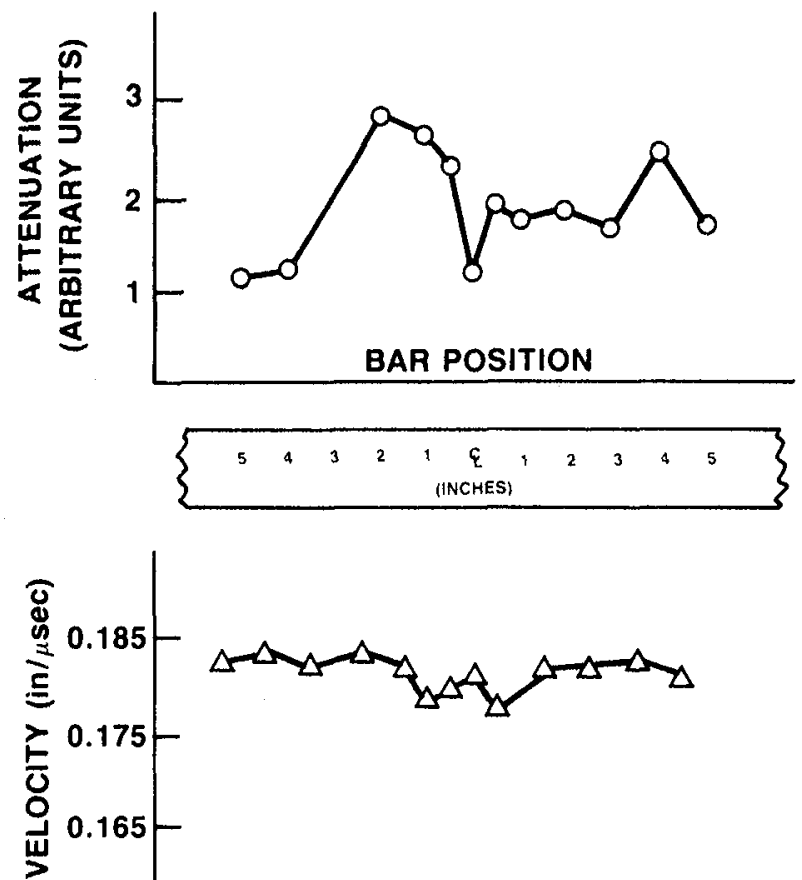

BAR POSITION

Fig 2 Velocity/attenuation characterization of each specimen.

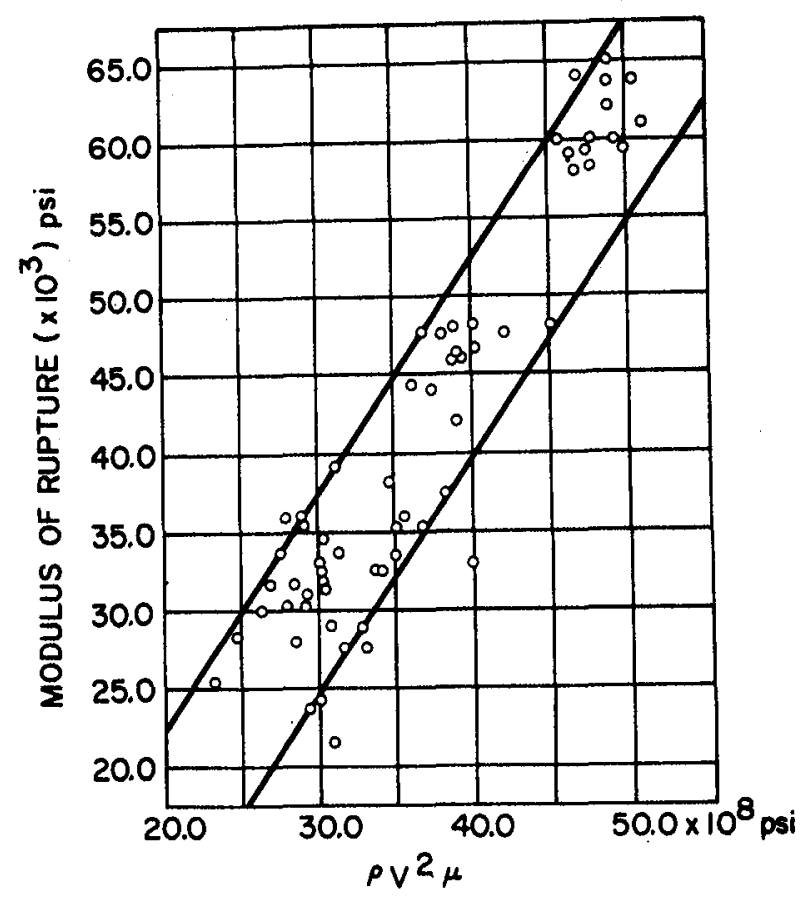

Fig 4 Modulus of rupture correlation in cast iron. 


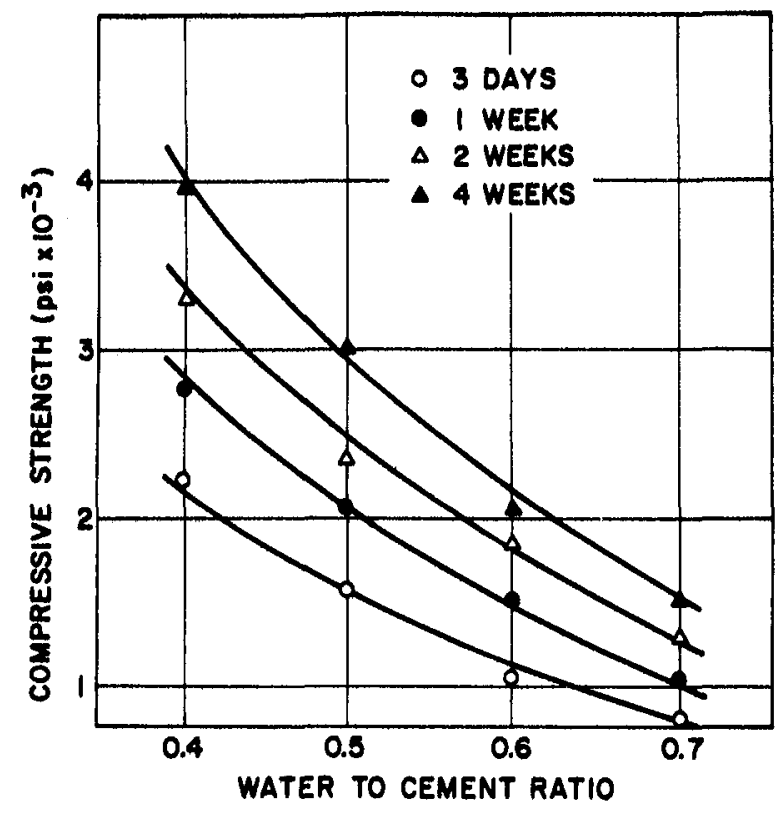

Fig 5 Concrete compressive strength vs water to cement ratio

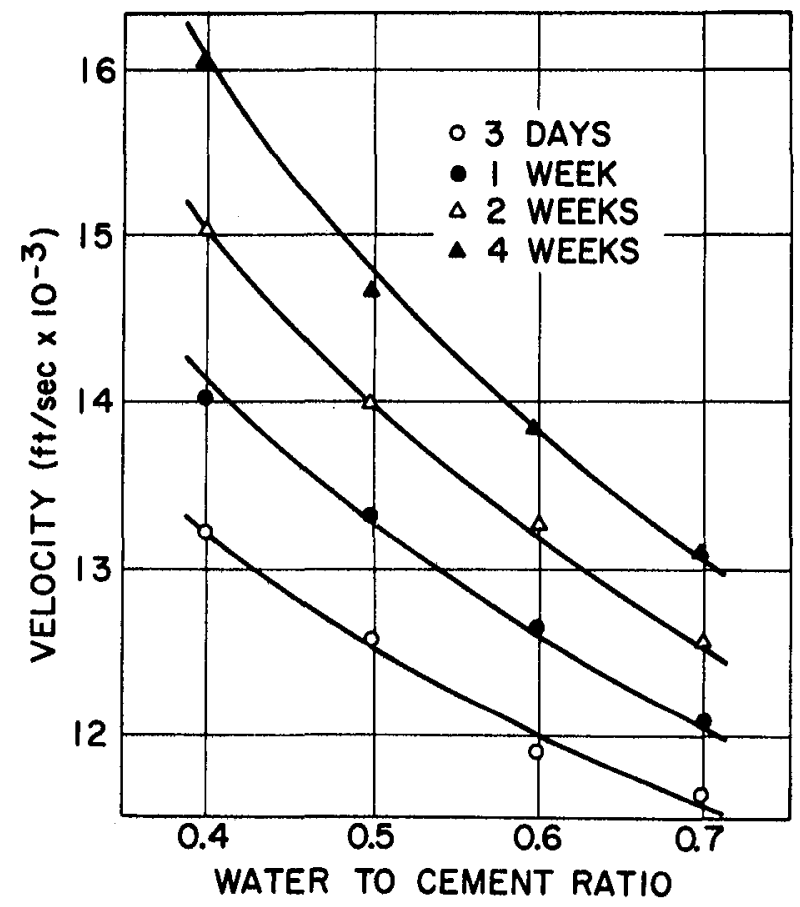

Fig 7 Compressive strength/velocity correlation in concrete.

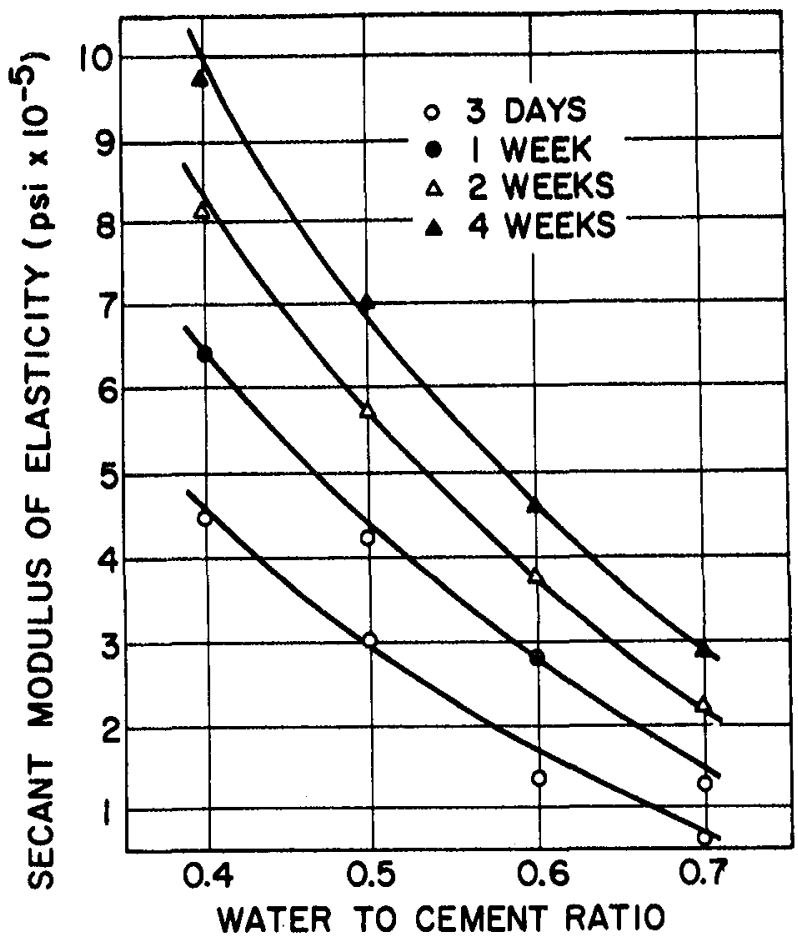

Fig 6 Concrete secant modulus vs water to cement ratio

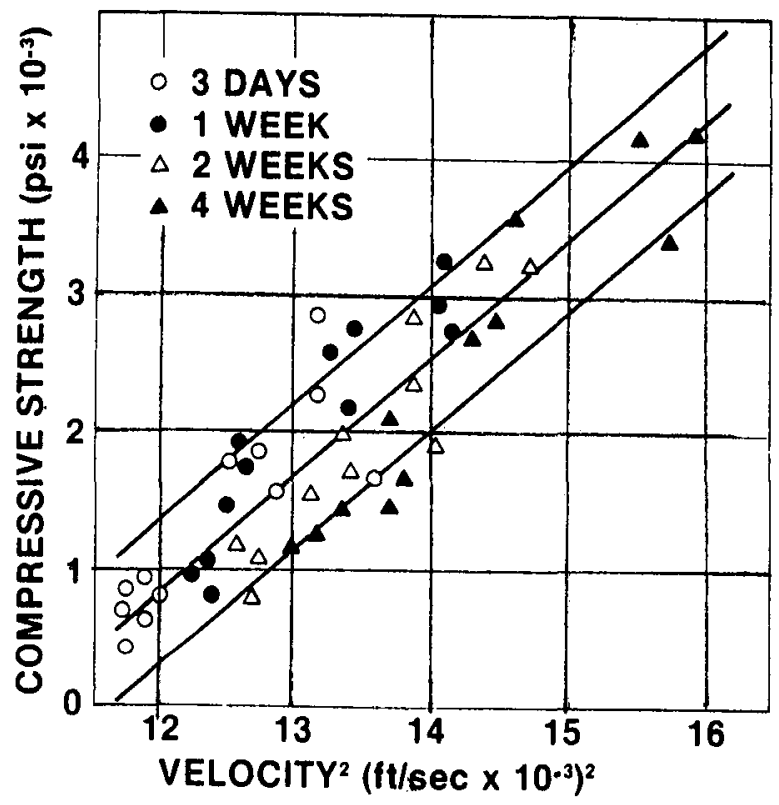

Fig 8 Secant modulus/velocity correlation in concrete. 


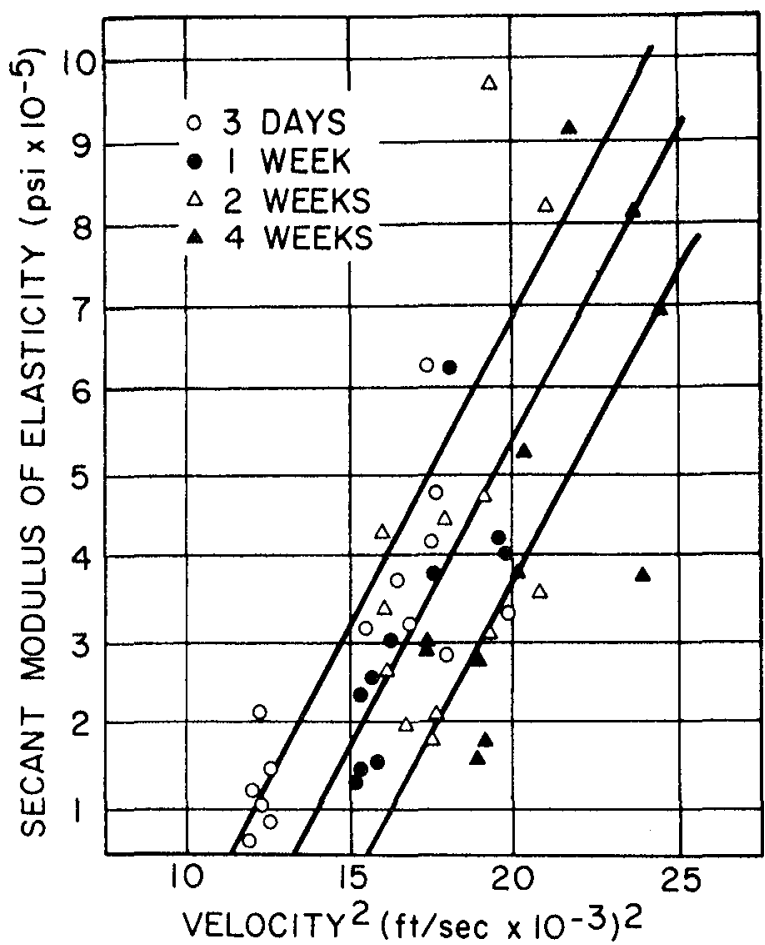

Fig 9 Secant modulus/velocity correlation in concrete.

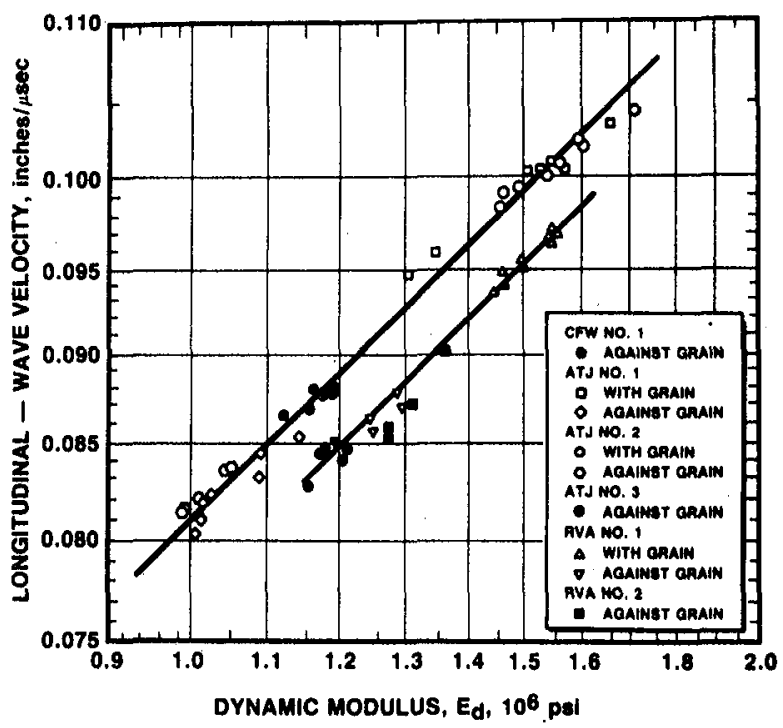

Figd Velocity/modulus correlations in graphite. Densities are $1.72 \& 1.85$ $\mathrm{gm} / \mathrm{cm}^{3}$ for the top and bottom plots, respectively,

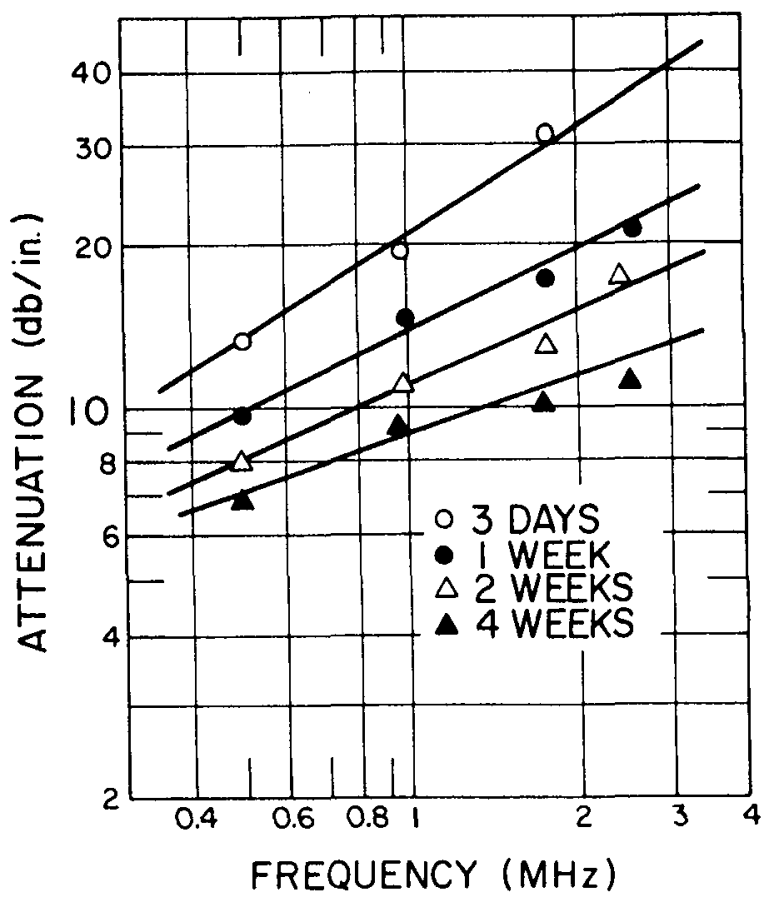

Fig 10 Attenuation monitoring of cure in concrete with a water to cement ratio of 0.5 .

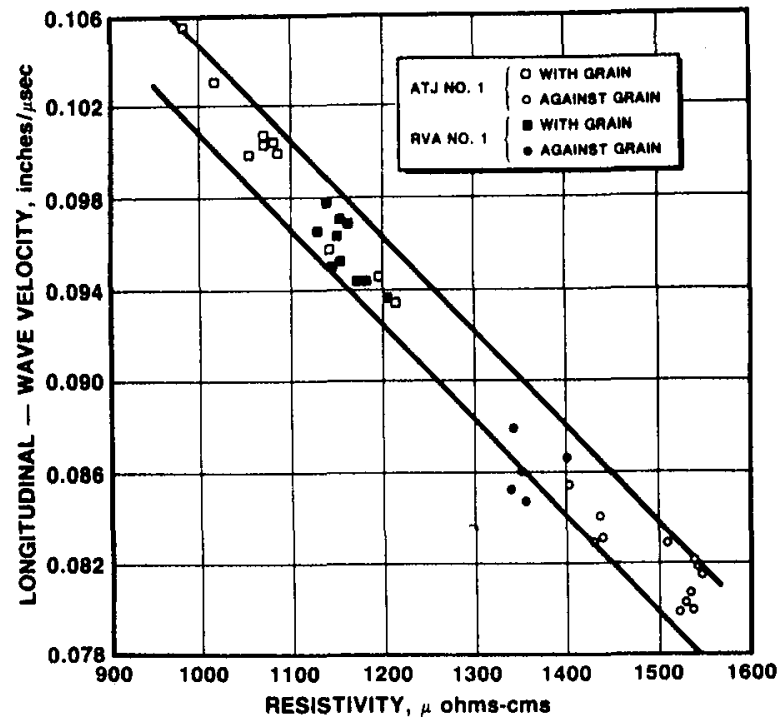

Fig 12 Velocity/resistivity correlation in graphite. 


\begin{tabular}{|c|c|c|}
\hline $\begin{array}{c}\text { WAYELENGTH TO } \\
\text { GRAIN DIAMETER } \\
\text { RANGE }\end{array}$ & MECHANISH & ATTENUATION \\
\hline$\lambda>>\bar{D}$ & RAYLEIGH & $\overline{\left(\frac{\Delta K}{K}\right)^{2}} \bar{D}^{3} \mathrm{Q}^{4}$ \\
$\lambda-\bar{D}$ & PHASE & $\overline{\left(\frac{\Delta K}{K}\right)^{2}} \bar{D}^{2}$ \\
$\lambda<<\bar{D}$ & DIFFUSION & $\overline{\left(\frac{\Delta K}{K}\right)^{2}} \frac{1}{\bar{D}}$ \\
\hline
\end{tabular}

Fig 13 Ultrasonic scattering losses.

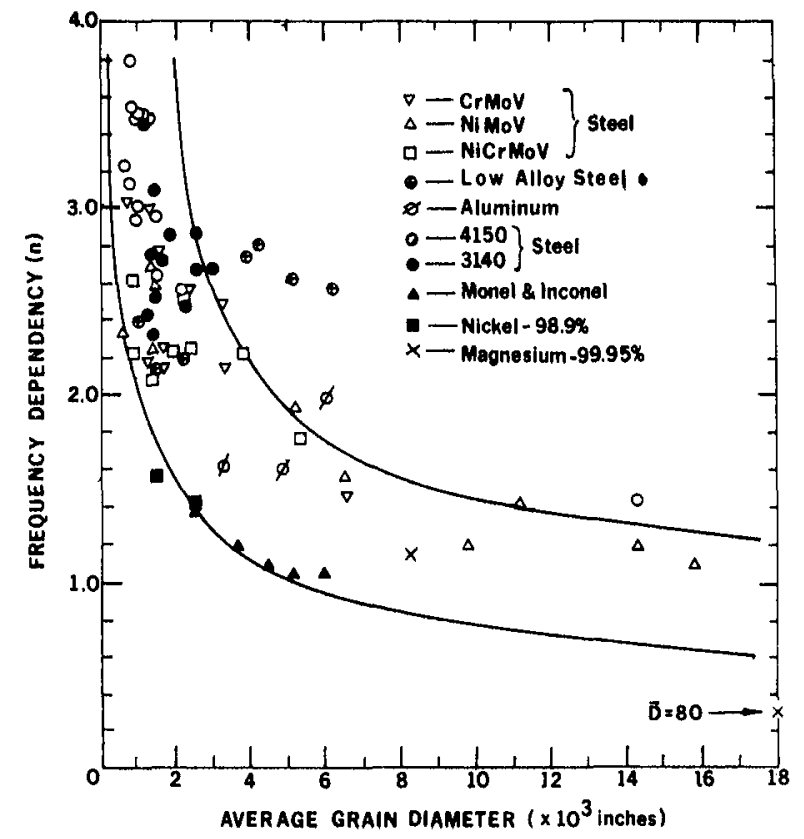

Fig 15 Frequency dependency as function of grain size.

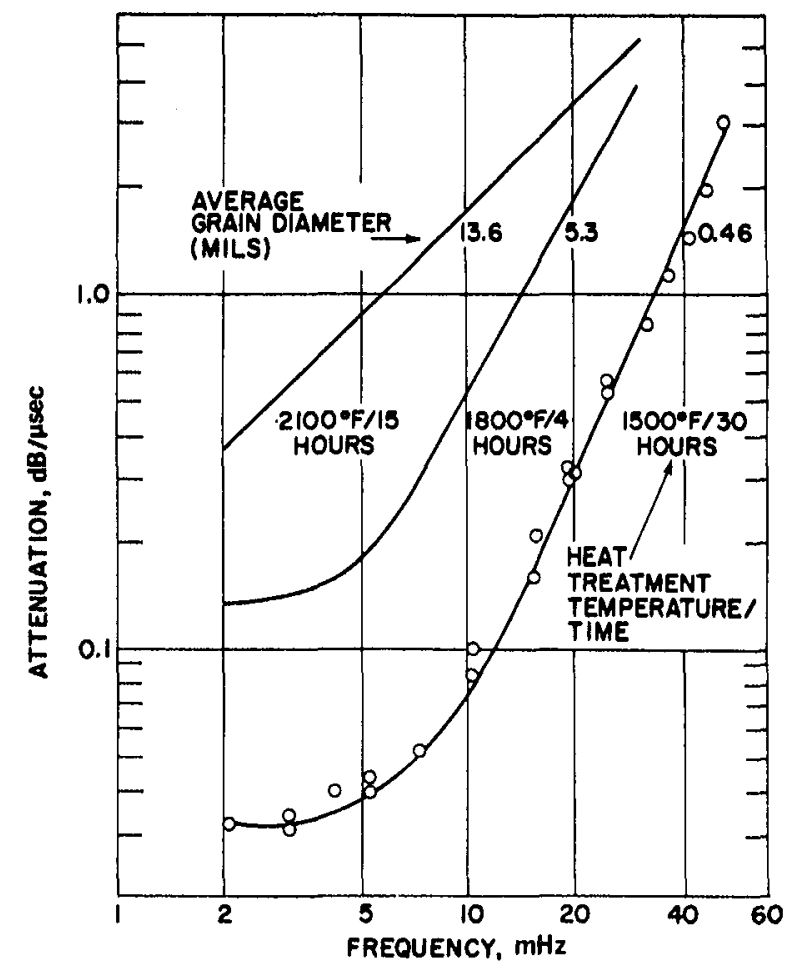

Fig 14 Attenuation/grain size effects.

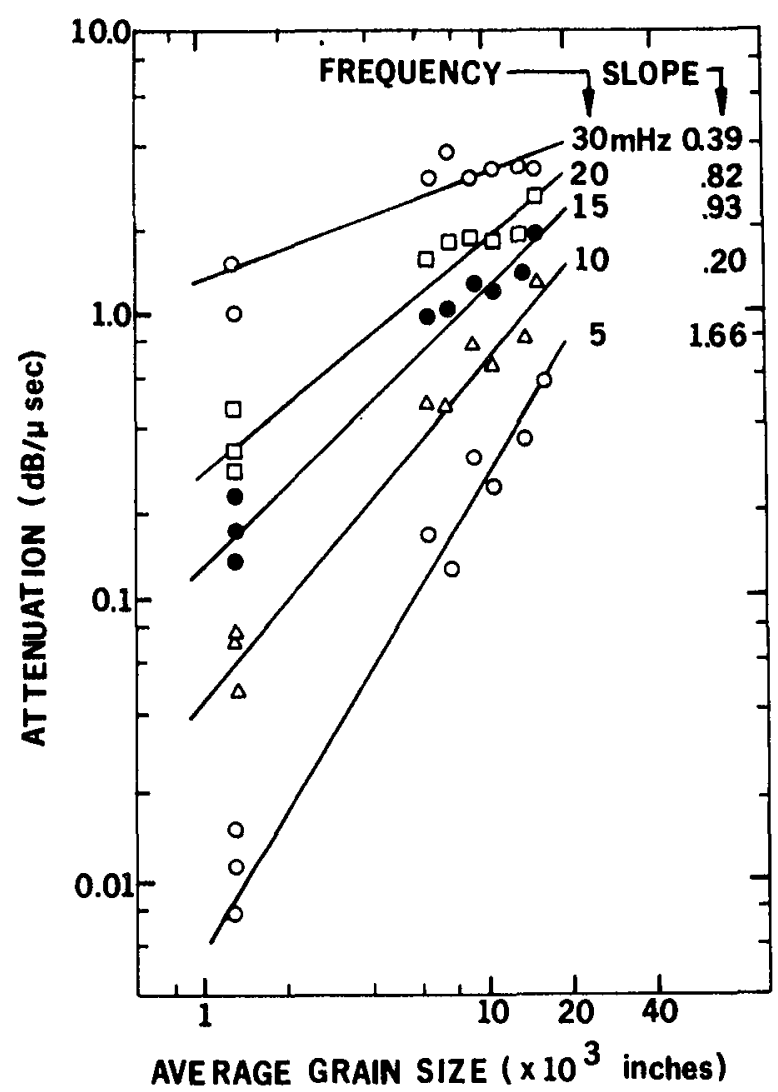

Fig 16 Cross plot of attenuation/grain size data typified by Fig 14. 


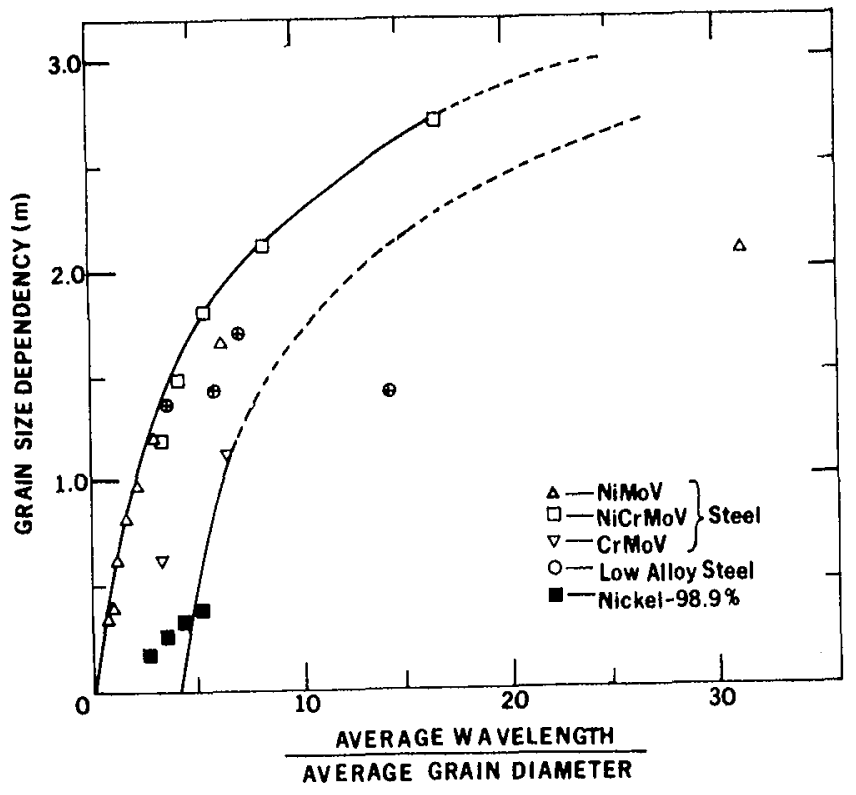

Fig 17 Grain size dependency of

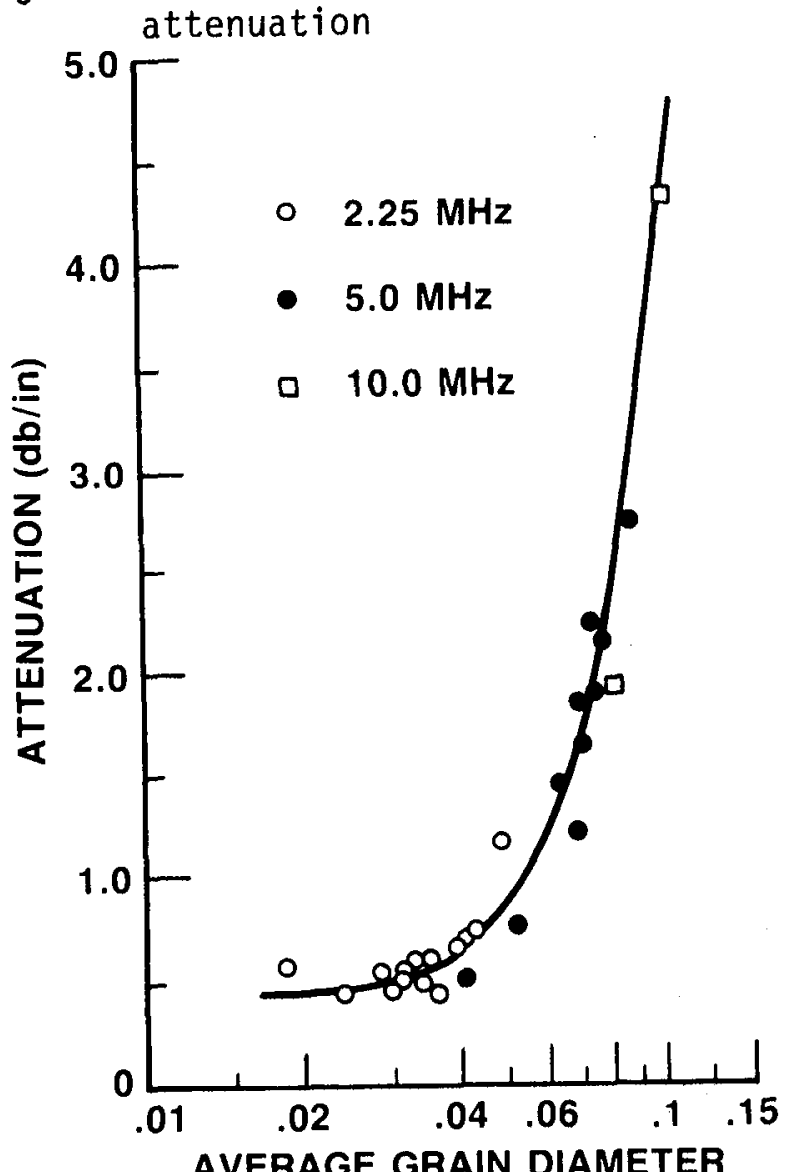

AVERAGE GRAIN DIAMETEB.

\section{WAVELENGTH}

Fig 19 Attenuation as a function of the ratio of the average grain size to wavelength.

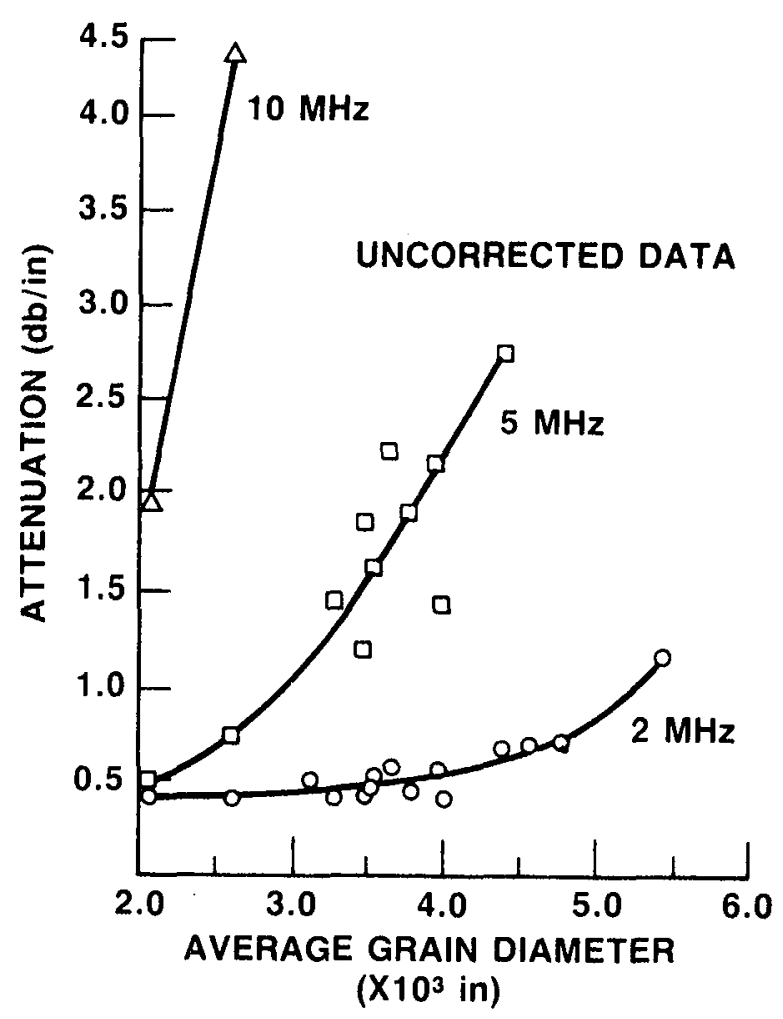

Fig 18 Attenuation/grain size for a Timkin alloy $(6-26-16)$

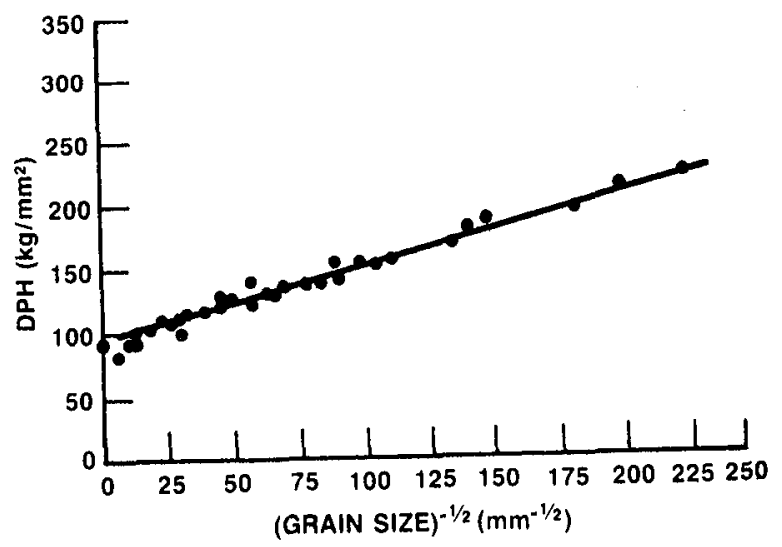

Fig 20 Hardness vs grain size in in titanium. After Armstrong (6) 


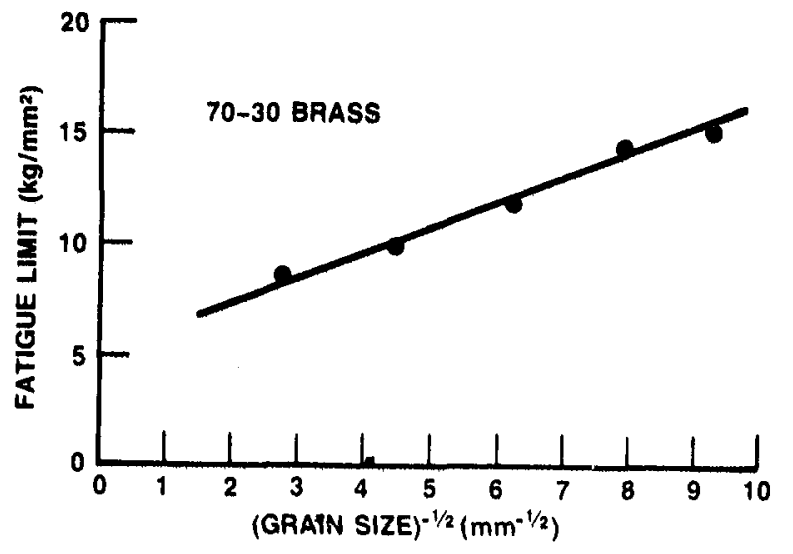

Fig 21 Fatique limit in $70-30$ brass. After Sinclair and Craig (7).

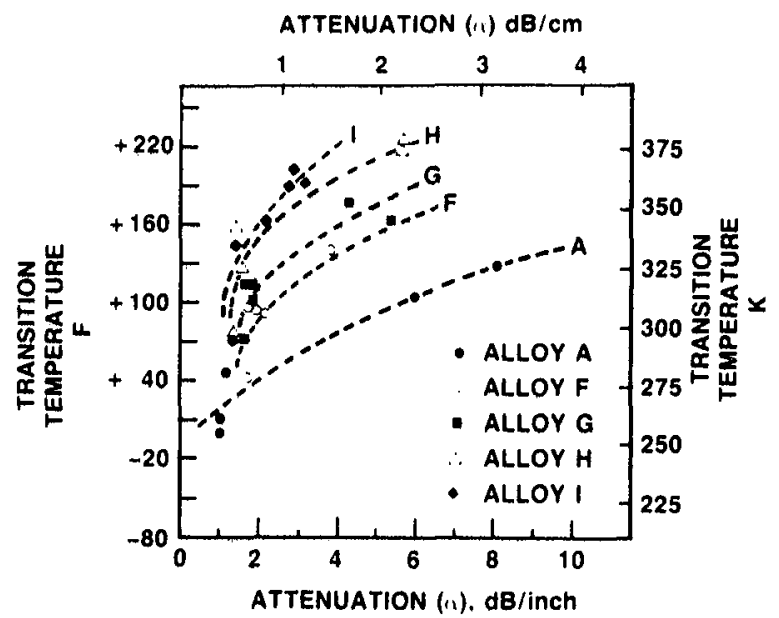

Fty 23 Attenuation monitoring of the transsition temperature of carbon steels. Carbon increases the transition temperature. After Klineman et al (9)

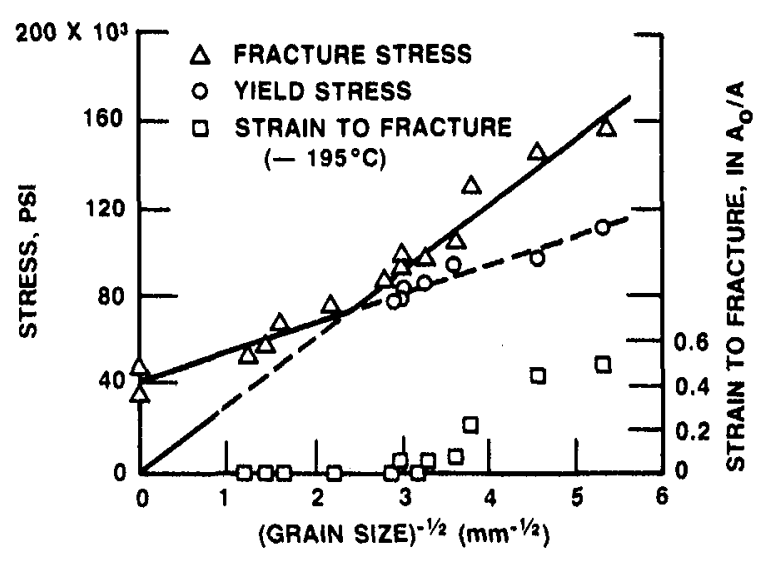

Fig 22 Influence of grain size on various properties of low carbon steels. After Low (8)

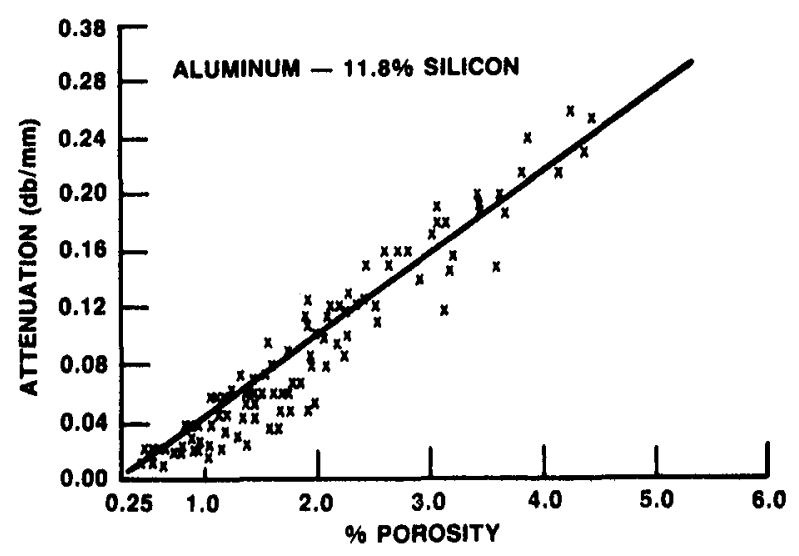

Fig 24 Attenuation/porosity correlations. After Chinnathambi and Prabhak (10). 


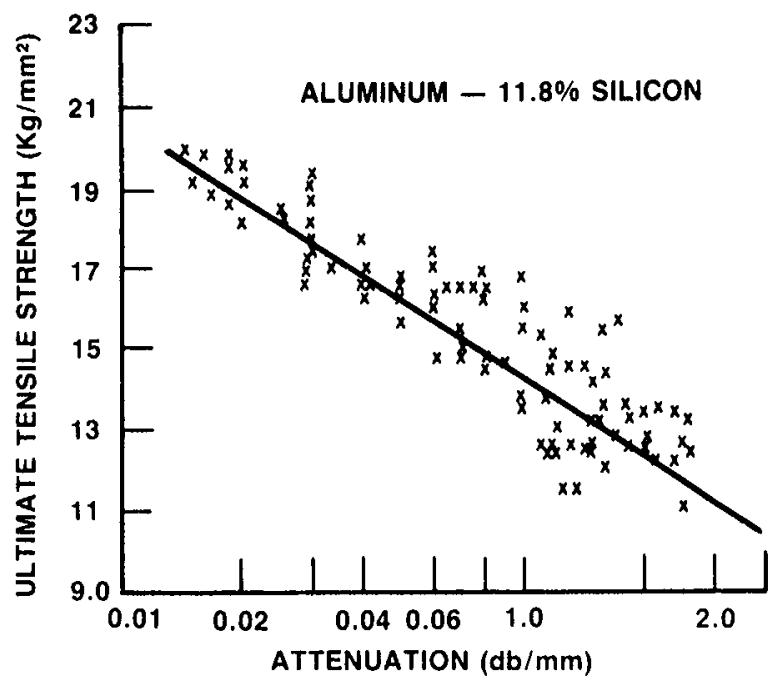

Fig 25 Attenuation monitoring of ultimate tensile strength. After Chinnathambi and Prabhak (10)

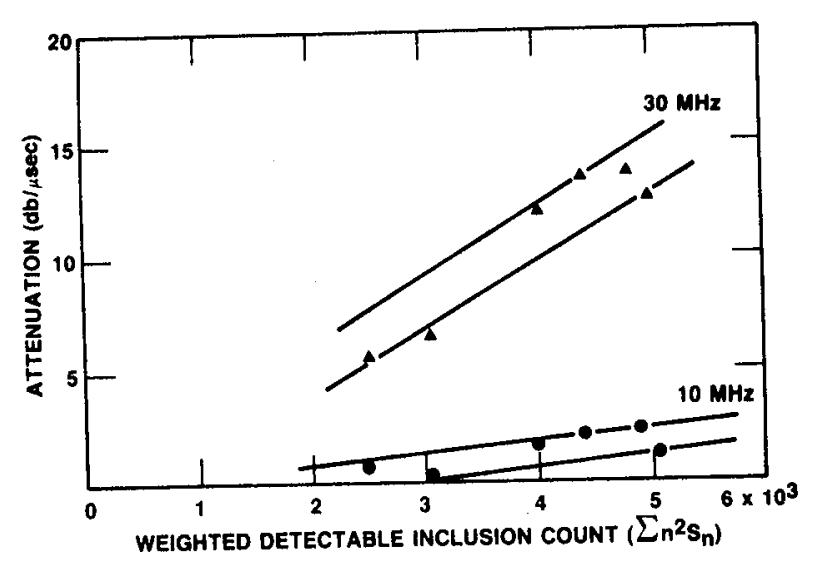

Fig 26 Attenuation monitoring of inclusion content in stainless steel.

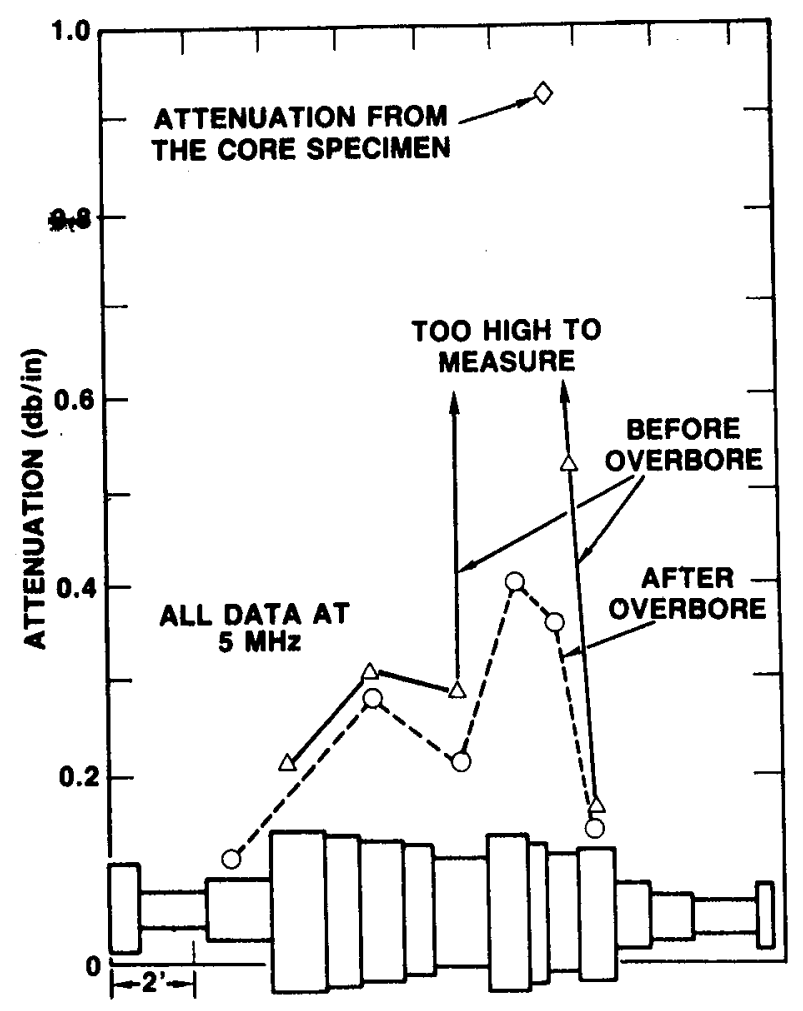

Fig 27 Monitoring of inclusion content in large rotor forgings. 